radiological investigations procedures before and after MRE was calculated. Clinician recorded Montreal classification 3 months before MRE and post-MRE was recorded in relation to changes to classification in extent $(\mathrm{L})$ and disease behaviour (B). Any change to management within 3 months following MRE was noted.

Results 31 patients were included in analysis. Male to female ratio was 1:2. Mean age at diagnosis of Crohn's disease was 17.9 years and at the point of MRE scan was 21.25 years. The mean duration of Crohn's disease follow-up pre-MRE was 2.6 years and post-MRE was 1.28 years. The indications included obstructive symptoms ( $n=14,45.1 \%)$, assessment of extent ( $n=15,48.3 \%)$, suspicion of internal fistula $(n=1,3.2 \%)$ and non-responsiveness to therapy $(n=1,3.2 \%)$. The findings included small bowel stricture (20), stricture with associated abscess (3), colonic stricture ( $\mathrm{n}=2)$, thickening (3), fistula (1) and gastric outlet obstruction (1). In 6 patients the MRE was normal. Following MRE 16 patients (51.6\%) had upgrading of disease behaviour (B) and 3 of them also had additional disease location (L). In 4 patients the MRE identified longer length of small bowel involvement than recorded before. The average radiation exposure pre-MRE was $7.47 \mathrm{mSv}$ and post-MRE was $1.56 \mathrm{mSv}$. In 4 patients the planned management was modified due to additional findings in MRE result.

Conclusion This study describes our experience in the use of MRE in a high risk group of adolescent and young adults with Crohn's disease. Application of specific referral criteria and patient selection may increase pick up rate, improve clinical utility by altering disease classification and extent, reduce radiation exposure and potentially reduce costs. Longer follow-up of a larger cohort of patients following introduction of $M R E$ in a hospital is needed to confirm reduction in radiation exposure in this group.

Competing interests None.

\section{PTH-055 MAGNETIC RESONANCE ENTEROGRAPHY IN ADOLESCENTS AND YOUNG ADULTS WITH CROHN'S DISEASE}

doi:10.1136/gut.2011.239301.456

P Kumar, 1,* A Razack, ${ }^{1}$ D Ashok, N Rao, ${ }^{1}$ J Cast, ${ }^{1}$ A Azaz, ${ }^{1}$ S Sebastian' ${ }^{1}$ Hull \& East Yorkshire NHS Trust, Hull, UK

Introduction MRE has been increasingly used for evaluation of known and suspected Crohn's disease patients but variable availability and high cost remains a problem in some areas. Young patients with Crohn's disease are reported to be particularly at higher risk of receiving high doses of radiation. There is limited data on the use of MRE in this group. The authors aimed to asses the effectiveness of use of MRE in adolescents and young adults based on select criteria used in our institution.

Methods Patients with known Crohn's disease diagnosed between 10 and 30 years undergoing MRE during a 2-year period were included. All had full endoscopic assessment and were classified based on Montreal classification. Demographics and clinical parameters were recorded. Actual radiation exposure in DAP (Dose Area Product) and milliseverts (mSv) recorded in RadCentre during procedure of all relevant 